\title{
Spectroscopy of gadolinium ion and disadvantages of gadolinium impurity in tissue compensators and collimators, used in radiation treatment planning
}

\author{
M. Maqbool ${ }^{\mathrm{a}, *}$ and Iftikhar Ahmad ${ }^{\mathrm{b}}$

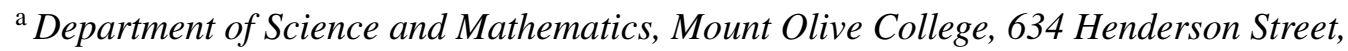 \\ Mount Olive, NC 28365, USA \\ ${ }^{\mathrm{b}}$ Department of Physics \& Astronomy, Louisiana State University, 202 Nicholson Hall, \\ Tower Dr. Baton Rouge, LA 70803-4001, USA
}

\begin{abstract}
Intense ultraviolet emission from gadolinium doped amorphous aluminum nitride thin films deposited on Si (111) substrate is studied with cathodoluminescence and photoluminescence. The purpose of the study is to find the merits or demerits of gadolinium ions if added intentionally or present as unintentionally added impurity in tissue compensators or collimators in radiation treatment planning. These films are deposited by reactive sputtering at liquid nitrogen temperature, using $100-$ $200 \mathrm{~W}$ RF power, 5-8 mTorr nitrogen, and a metal target of aluminum and gadolinium. Thermal annealing was performed at a temperature of $900^{\circ} \mathrm{C}$. A sharp ultraviolet peak is observed at $314 \mathrm{~nm}$ corresponding to ${ }^{6} \mathrm{P}_{7 / 2} \rightarrow{ }^{8} \mathrm{~S}_{7 / 2}$ transition. The ultraviolet emission is intense enough to harm human tissues if it is used as tissue compensator. Intense ultraviolet emission is observable even if the concentration of gadolinium is less than $0.5 \%$. Thermal annealing further enhances the intensity of ultraviolet emission, indicating that longer use of such tissue compensators or collimators containing gadolinium ions will provide more harm and damage to human body. Radiation Therapists, Oncologist and industries making tissue compensators and collimators are strongly suggested to test any compensator or collimator for gadolinium impurities.
\end{abstract}

Keywords: Gadolinium, cathodoluminescence, photoluminescence, ultraviolet emission, tissue damage, tissue compensator

\section{Introduction}

Radiation oncology is the specialty of medicine that utilizes high-energy ionizing radiation in the treatment of malignant tumors and certain non-malignant conditions [1-3]. Supply of appropriate and uniform dose to a tumor with minimum possible damage to normal tissues is a key factor in such treatment planning $[1,2,4]$. Excessive irradiation, however, leads to the destruction of normal tissues as well as the cancer, whilst inadequate dosage fails to kill the cancer cells, so that after a time they recover from such damage as they have sustained, regain their vitality, and start to multiply again $[2,5]$. Thus an optimum dose is required to treat a specific tumor. Similarly surface irregularities, organ movement and

\footnotetext{
${ }^{*}$ Corresponding author: M. Maqbool, Department of Science and Mathematics, Mount Olive College, 634 Henderson Street, Mount Olive, NC 28365, USA. Tel.: +1 (919) 6587498; Fax: +1 (919) 6587898; E-mail: mmaqbool@ moc.edu
} 
tissue inhomogeneities cause varying source to surface distance (SSD) over the treatment field, resulting in a non-uniform radiation dose within the tumor volume. Such irregularities and inhomogeneities causing complications $[1,2,4-7]$. Different techniques are used to overcome these problems. One of those important techniques is the use of tissue compensators [1,5,8,9]. A number of new materials, not necessarily tissue equivalent, are used to design various kinds of compensators in different ways and shapes [1,5,9-11]. Commonly, alloys are used in making compensators [5]. Various properties and characteristics of such compensators, absorption and attenuation coefficients and transfer functions, need to be determined and studied before their practical use [9]. Dose distribution and scattering is one of those important properties $[4,6,9]$. Another important area which needs attention is the ultraviolet emission from certain materials used or come as unintentionally added impurity in these alloys. For example $\mathrm{Ho}^{+3}$, $\mathrm{Pr}^{+3}$ and $\mathrm{Tm}^{+3}$ are ultraviolet emitters and when exposed to electron or photon beam they emit ultraviolet radiations [12-14]. If alloys containing one of these materials, either added intentionally or coming unintentional as impurity, is used as tissue compensator then the characteristic ultraviolet emission can cause damage to normal tissues [16,17]. One of those ultraviolet emitters is gadolinium $\left(\mathrm{Gd}^{+3}\right)$.

In the present work thin film of Gd doped aluminum nitride (AIN) alloy is deposited and studied for its characteristic emissions when exposed to an electron and photon beams using cathodoluminescence (CL) and photoluminescence (PL) respectively. Both CL and PL spectra are obtained for the as-deposited and thermally annealed films. This emission occurs as a result of transition from the first excited ${ }^{6} \mathrm{P}_{7 / 2}$ state of gadolinium ion to its ground ${ }^{8} \mathrm{~S}_{7 / 2}$ state.

\section{Methods and materials}

Thin films of amorphous AlN:Gd were prepared at $77 \mathrm{~K}$ by radio frequency (rf) magnetron sputtering of an aluminum target of $99.999 \%$ purity in a pure nitrogen atmosphere. Doping of the thin film with gadolinium $(\mathrm{Gd})$ was accomplished by drilling a small hole $(0.5 \mathrm{~cm}$ diameter) in the aluminum target ( $4.2 \mathrm{~cm}$ diameter) and placing a slug of $\mathrm{Gd}$ in the hole. Gd was then co-sputtered with the aluminum. The rf power was varied between 100 and 200 watts. All films were deposited on p-silicon (111) substrates. The background pressure in the chamber was $<3 \times 10^{-5}$ Torr. Liquid nitrogen was used to keep the temperature of the film at $77 \mathrm{~K}$. The metallic substrate holder was designed such that it was having a half inch diameter cylindrical hole from the top. The substrate was pasted on the metal base of the holder below the liquid nitrogen. Liquid nitrogen was constantly poured in the holder to provide a constant low temperature to the substrate during film growth.

The as-deposited films were characterized for their characteristic emissions. The thickness of the film was between $300 \mathrm{~nm}$, measured with a quartz crystal thickness monitor in the growth chamber. X-rays diffraction (XRD) was used to determine the structure of the films. No diffraction peaks were observed, indicating that the as-deposited films were amorphous.

CL studies of the films were performed at room temperature in a vacuum chamber at a pressure of about $3 \times 10^{-6}$ Torr, which was maintained with an Alcatel CFF 450 turbo pump. Films were excited with electron beam energy of $2.85 \mathrm{kV}$ and beam current of $100 \mu \mathrm{A}$. Luminescence from the films was focused onto the entrance slit of a SPEX Industries double monochromator with gratings blazed at 500 $\mathrm{nm}$ and detected at a Thorn EMI fast high gain photomultiplier tube with a range of 200-900 nm. Thermal annealing was accomplished by placing the flat films in a tube furnace at $900^{\circ} \mathrm{C}$ in a nitrogen atmosphere for half an hour. 
PL measurements were made using a commercially available fluorimeter (Photon Technology International, model C-60SE) with a double emission monochromator. The detector in steady state PL experiments was a photon counting photomultiplier tube. Samples were immobilized in a specially made solid sample holder. Xenon lamp was used to illuminate and excite the film by photon beam of desired wavelength.

\section{Results}

Figure 1 shows the room temperature CL spectrum of gadolinium doped amorphous AlN in $300 \mathrm{~nm}$ to $800 \mathrm{~nm}$ range. This range covers a portion of ultraviolet region, the entire visible range and a part of infrared region of the spectrum. Strong and sharp peak at $314 \mathrm{~nm}$ is obtained. This sharp peak results from transition between the first excited state ${ }^{6} \mathrm{P}_{7 / 2}$ and ground state ${ }^{8} \mathrm{~S}_{7 / 2}$ of $\mathrm{Gd}^{+3}$. No other strong peaks are observed which means other states are either not excited or are nonradiatively depopulated and Gd is ultraviolet emitter only.

Figure 2 shows the CL spectrum of thermally annealed gadolinium doped AlN film. Thermal annealing was accomplished by placing the flat films in a tube furnace at $900^{\circ} \mathrm{C}$ in a nitrogen atmosphere for 30 minutes. It is clear from Fig. 2 that thermal annealing has strongly affected the ultraviolet emission from $\mathrm{Gd}$ and the $314 \mathrm{~nm}$ peak more intense as compared to the same peak without thermal annealing. It is evident from the figure that the intensity of $314 \mathrm{~nm}$ peak after thermal annealing has increased by a factor more than $100 \%$. Clearly it means that heating of Gd alloys will enhance the ultraviolet emission from Gd.

Figure 3 represents the PL spectrum of gadolinium doped amorphous aluminum nitride. The as deposited film was excited at $295 \mathrm{~nm}$ and the emission spectrum was obtained from $305 \mathrm{~nm}$ to $550 \mathrm{~nm}$. An intense but broad emission from $308 \mathrm{~nm}$ to $340 \mathrm{~nm}$ with a width of about $32 \mathrm{~nm}$ was obtained. This result is quite matching with the CL spectrum of the same film. In CL spectrum we obtain a sharp and high intense peak at $314 \mathrm{~nm}$. However in PL spectrum due to the broadness of the emission band we see an emission range from $308 \mathrm{~nm}$ up to $340 \mathrm{~nm}$.

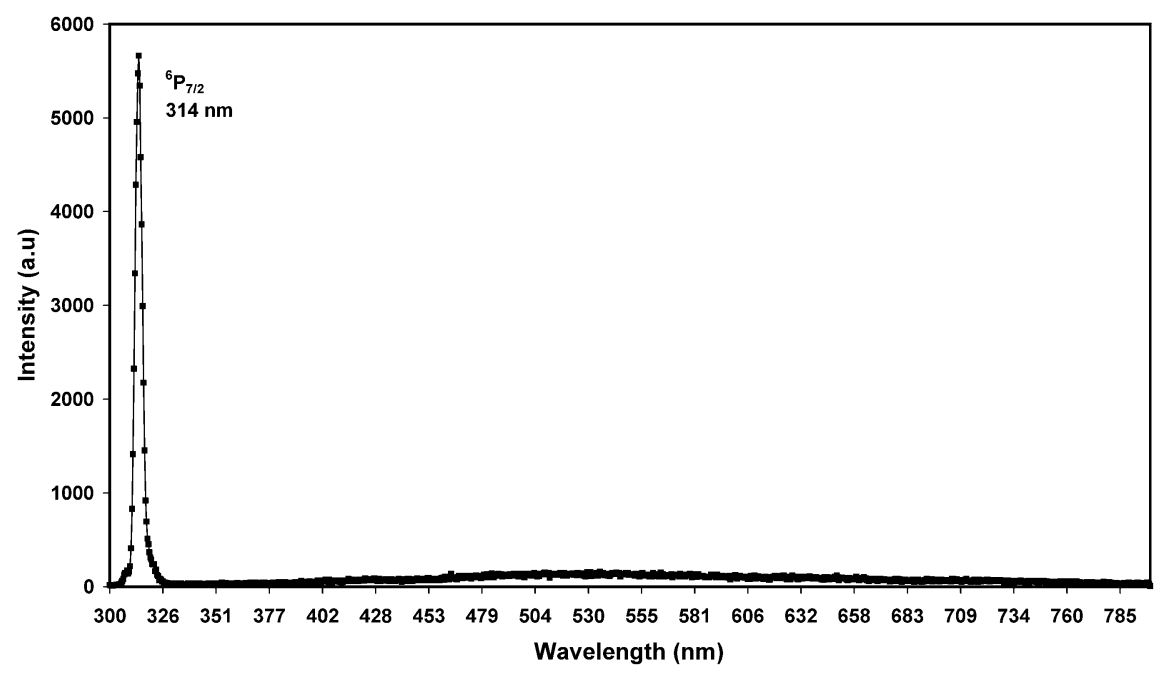

Fig. 1. Ultraviolet emission from Gd doped amorphous AlN films. 


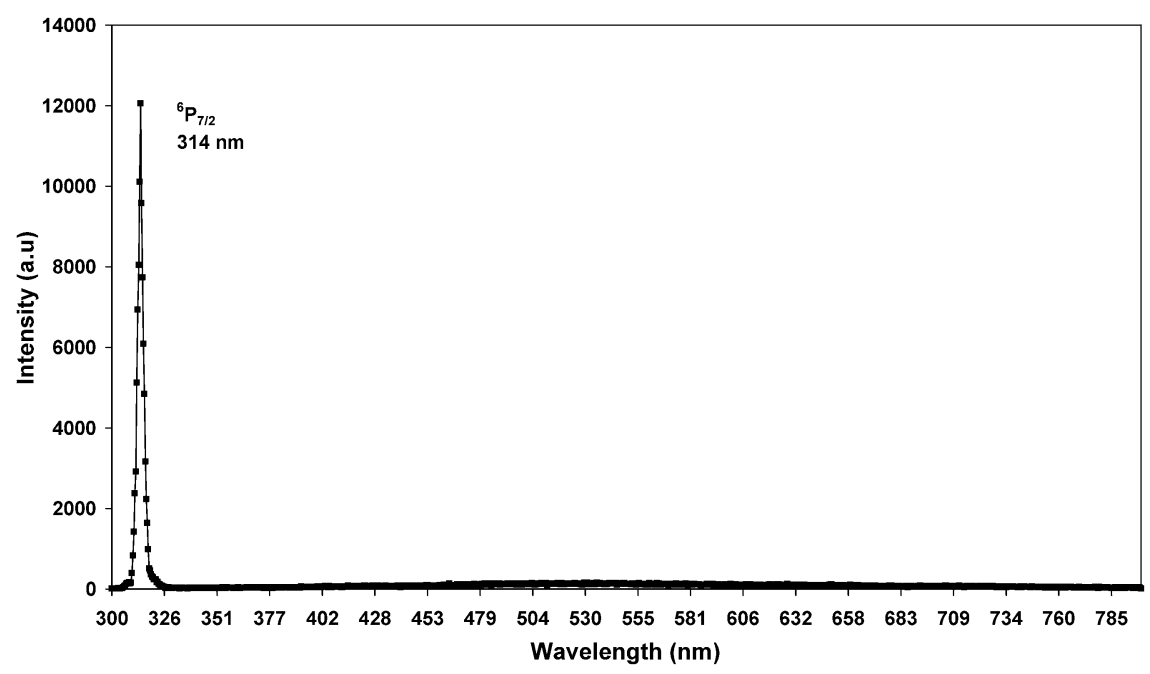

Fig. 2. Emission from AlN:Gd film thermally annealed at $900^{\circ} \mathrm{C}$ for 30 minutes.

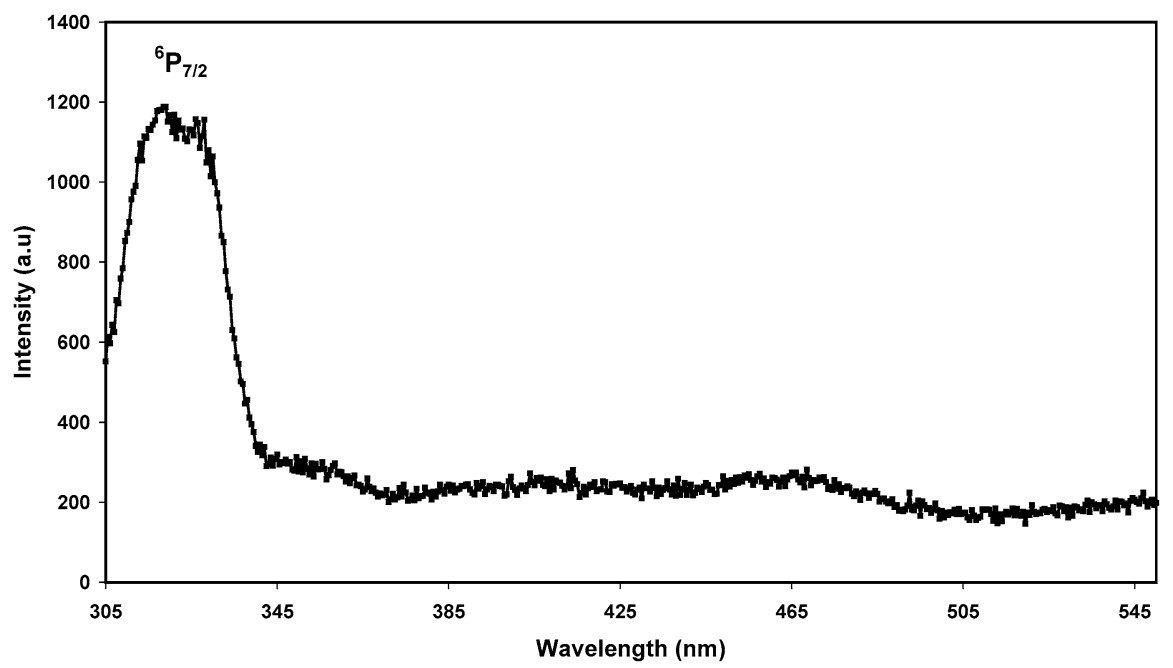

Fig. 3. PL spectrum of Gd doped AlN in $305 \mathrm{~nm}$ to $550 \mathrm{~nm}$ range excited by $300 \mathrm{~nm}$ photon beam.

\section{Discussion}

The results obtained in Fig. 1 indicate that alloys with Gd impurity are not a good choice to make a tissue compensator and a collimator from, for radiation treatment. Since the supply of accurate and precise dose to a tumor is one of the key factors in cancer treatment and hence more or less than the appropriate dose supplied to a tumor will cause complications. If $\mathrm{Gd}$ is added intentionally or present as an unintentionally added impurity in a tissue compensator or a collimator then it will emit ultraviolet radiations when exposed to photon or electron beam obtained from linear accelerator or any other source. Those ultraviolet radiation will hit the normal tissues and some times tumor (depending on the location of tumor), and will create complications in the tumor control and protection of healthy tissues. These complications will arise in three different ways. 
First, the healthy tissues and tumor will receive more dose than the estimated one because Gd ion will emit ultraviolet radiation that will come in addition to the dose supplied by the source. Second, in case of electron beam therapy two different kind of radiation, the electron beam and ultraviolet rays, will hit the body and hence calculations for dose supply, estimate of damage to normal tissue, especially the skin and supply of dose to tumor will need modification and new calculations will be needed keeping both type of radiation in view. Third, in case of photon beam therapy though the nature of radiation will be the same but it is not necessary that the energy and wavelength of the radiation dose obtained from the source will be the same as of the ultraviolet radiation emitted from Gd. Changing the energy and wavelength of photon beam brings changes in some important characteristics of the beam, like linear energy transfer, which brings different effects on human body, like ultraviolet rays can mainly damage the skin while gamma rays can also provide damage to the tissues beyond the skin. Thus two different kind of effect from the same nature of radiation will be kept in mind.

Further, Fig. 2 shows that when Gd doped AlN films are heated above room temperature then the emission of ultraviolet radiation gets more intense. This fact makes us further cautious about the presence of Gd impurity in tissue compensators or collimators because if such kind of compensator or collimators are used for long time in one time use then longer time exposure to radiation beam will heat this compensator or collimator and the ultraviolet emission of Gd will get highly intense. This more intense ultraviolet emission will cause more damage to the normal tissues.

Overall Gd alloys will bring quantitative as well as qualitative changes to the supply of dose to a tumor and will damage the normal tissues if added intentionally or present as an unintentionally added impurity in a tissue compensator or a collimator.

However some materials can be used as natural filters for ultraviolet radiation coming from $\mathrm{Gd}$. In the present work an alloy of Gd doped in AlN is studied. We have already reported that the wide bandgap $6.2 \mathrm{eV}(190 \mathrm{~nm})$ of AlN is transparent to the ultraviolet radiation with wavelength more than this bandgap [13]. On the other hand the bandgap of gallium nitride $(3.4 \mathrm{eV}$ or $352 \mathrm{~nm})$ absorbs ultraviolet radiation of wavelength shorter than $352 \mathrm{~nm}$. The best example is the emission of $335 \mathrm{~nm}$ ultraviolet radiations from praseodymium when praseodymium is doped in AlN [13]. We have also studied the emission from praseodymium when doped in gallium nitride $(\mathrm{GaN})$ and found that there is no emission of ultraviolet rays of wavelength $335 \mathrm{~nm}$ from praseodymium when doped in GaN $[13,18,19]$. Clearly the bandgap of $352 \mathrm{~nm}$ have absorbed the energetic ultraviolet radiation of wavelength $314 \mathrm{~nm}$ from Gd.

Thus GaN can be used as an ultraviolet filter for the ultraviolet emitter Gd ion if present as impurity in tissue compensators or collimators. Further GaN or any other material with band gap equal to or lower (in energy) than GaN bandgap can be used as an ultraviolet filter for any treatment or shielding application.

\section{Conclusion}

In conclusion, gadolinium doped amorphous aluminum nitride thin films deposited by rf magnetron sputtering were studied for its luminescence by cathodo- and photoluminescence. It was observed that Gd is a strong ultraviolet emitter in $314 \mathrm{~nm}$ when exposed to an electron or photon beam. Thermal annealing enhances the intensity of this ultraviolet emission by more than 100\%. Ultraviolet radiations are very dangerous to health and can damage human body. If $\mathrm{Gd}$ ion is added intentionally or present as unintentionally added impurity in tissue compensator or collimator then complications will arise due to the ultraviolet radiation from Gd when exposed to electron or photon beam, even if the concentration 
of $\mathrm{Gd}$ is as minute as less than $0.5 \%$. However $\mathrm{GaN}$ can be used as a natural filter to absorb the ultraviolet radiation of Gd. Radiation Therapists, Oncologist and industries making tissue compensators and collimators are strongly suggested to test any compensator or collimator for gadolinium impurities.

\section{References}

[1] M.R. Mosbacher, C.S. Wuu, G.J. Kutcher and S.R. Isaacson, A comparison of three dimensional conformal radiation therapy vs. intensity modulated radiation therapy and coplanar vs. non coplanar beam arrangements in the treatment of skull-base meningioma, Int. J. Radiat. Oncol. Biology \& Physics, 54(2, Suppl. 1) (2002), 322-332.

[2] F.M. Khan, The Physics of Radiation Therapy, Williams and Wilkins, London, 1984.

[3] C.K. Bomford, Text-Book of Radiotherapy, 5th edn, Churchill Livingstone Medical Division of Longman Group, United Kingdom Ltd, 1993.

[4] F.H. Attix, Introduction to Radiological Physics and Radiation Dosimetry, Willey, New York, 1986.

[5] G.A. Cardarelli, S.N. Rao and D. Cail, Investigation of the relative surface dose from Lipowitz-metal tissue compensators for 24- and 6-MV photon beams, Med. Phys. 18(2) (1991), 282-287.

[6] J.E. Turner, Atoms Radiation and Radiation Protection, 2nd edn, Willey, London, 1995.

[7] K.K. Brock et al., Inclusion of organ deformation in dose calculations, Med. Phys. 30(3) (2003), 290-295.

[8] F. Ellis, Radiology 106 (1973), 191-194.

[9] M. Maqbool, J. Mech. Med. Biol. 4(3) (2004), 305-310.

[10] F.M. Kahn, V.C. Moore and D.J Burns, Radiology 96 (1970), 187-192.

[11] R.J. Boge et al., Tissue compensators for megavoltage radiotherapy fabricated from hollowed Styrofoam filled with wax, Radiology 111 (1974), 193-198.

[12] M. Maqbool, H.H. Richardson, P.G. van Patten and M.E. Kordesch, Luminescent holmium doped amorphous AlN thin films for use as waveguides and laser cavities, Mat. Res. Soc. Symp. Proc., Vol. 798, Materials Research Society, 2004, pp. 8.5.1-8.5.5.

[13] M. Maqbool, H.H. Richardson and M.E. Kordesch, Cathodoluminescence of praseodymium doped amorphous AlN, GaN and turbostratic BN, Mater. Res. Soc. Symp. Proc., Vol. 831, Materials Research Society, 2005, Article E8.12.1.

[14] H.J. Lozykowsky, W.M. Jadwisienczak and I. Brown, Photoluminescence and cathodoluminescence of GaN doped with Tb, Appl. Phys. Lett. 76 (2000), 861; 114 (1989), 127.

[15] M. Maqbool, Luminescence from thulium and samarium doped amorphous AlN thin films deposited by RF magnetron sputtering and the effect of thermal annealing on luminescence, Eur. Phys. J. Appl. Phys. 34 (2006), 31-34.

[16] H.R. Taylor et al., Effect of ultraviolet radiation on cataract formation, New Engl. J. Med. 319 (1988), 1429-1133.

[17] B.L. Diffey, Solar ultraviolet radiation effects on biological systems, Phys. Med. Biol. 36 (1991), 299-328.

[18] R. Birkhahn, M. Garter and A.J. Steckl, Red light emission by photoluminescence and electroluminescence from Pr-doped GaN on Si substrate, Appl. Phys. Lett. 74(15) (1999), 2161-2163.

[19] V.K. Tikhomirov, K. Lakoubovskii, P.W. Hertogen and G.J. Adriaenssens, Visible luminescence from Pr-doped sulfide glasses, Appl. Phys. Lett. 71(19) (1997), 2740-2742. 


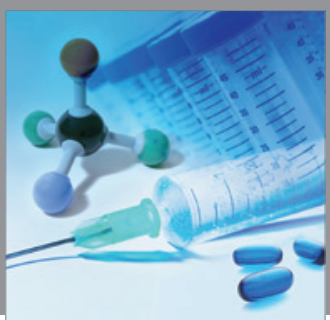

International Journal of

Medicinal Chemistry

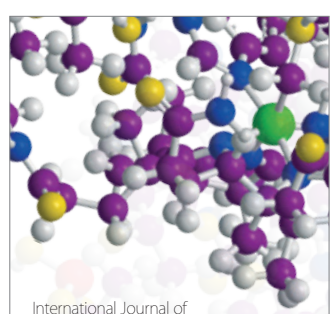

Carbohydrate Chemistry

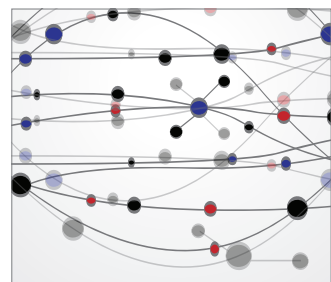

The Scientific World Journal
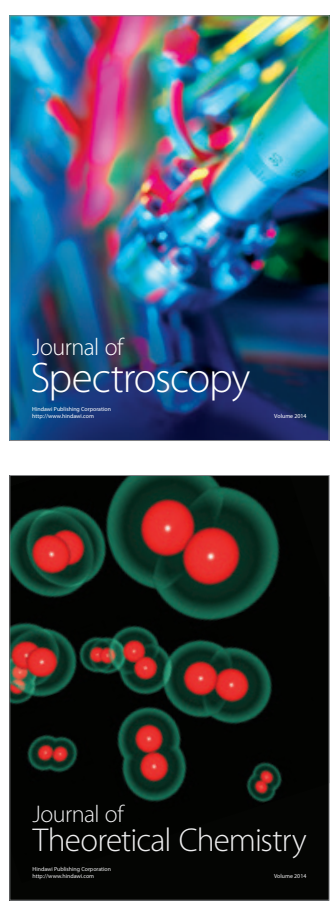
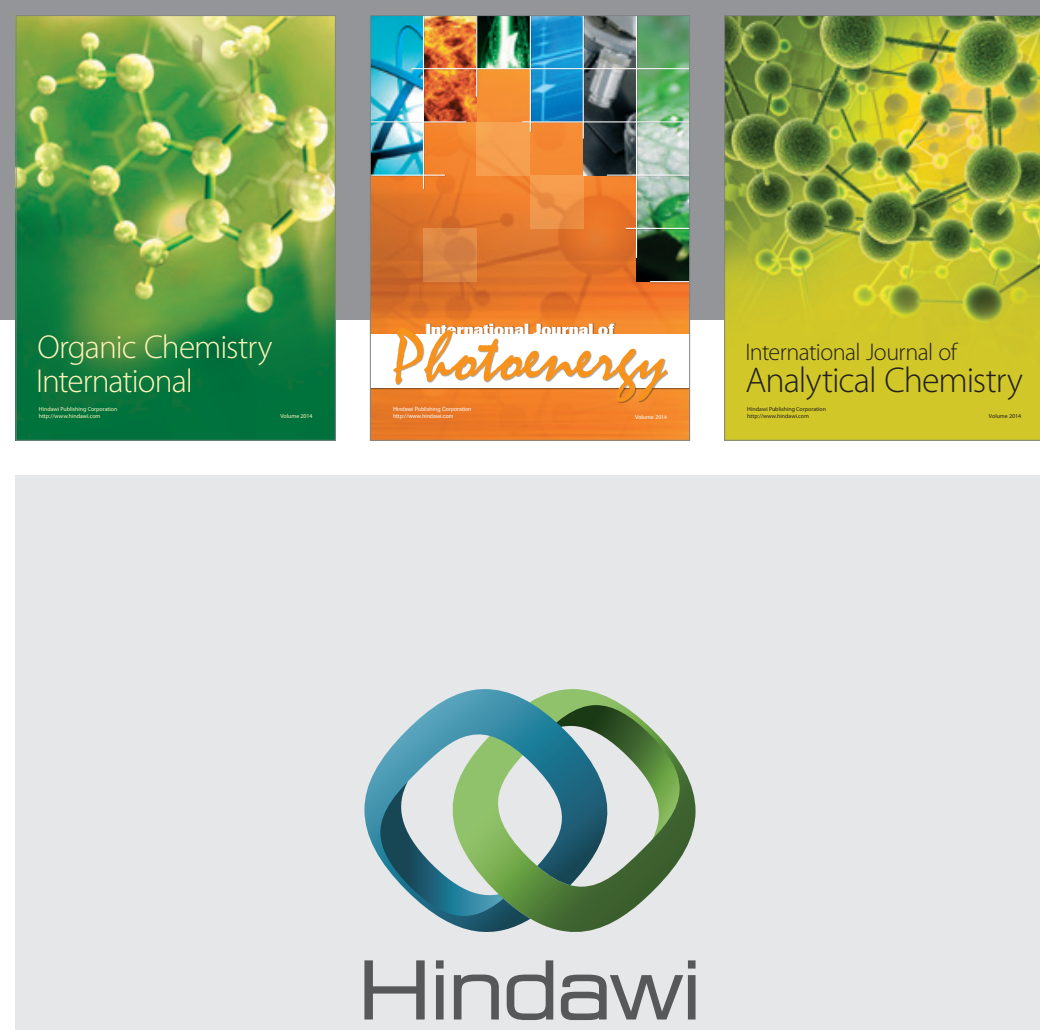

Submit your manuscripts at

http://www.hindawi.com
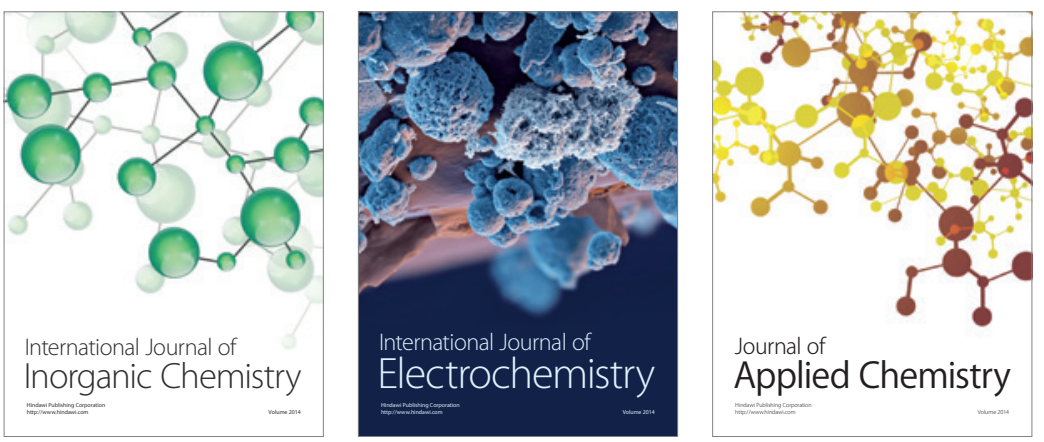

Journal of

Applied Chemistry
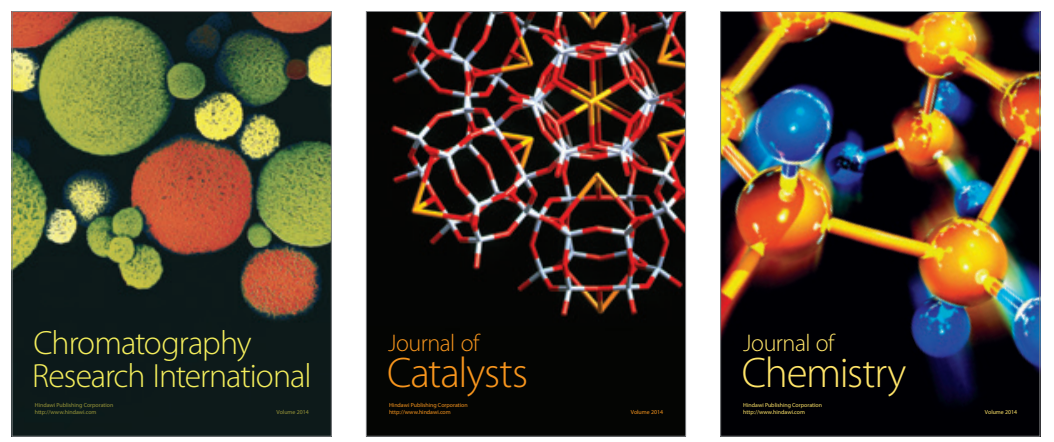
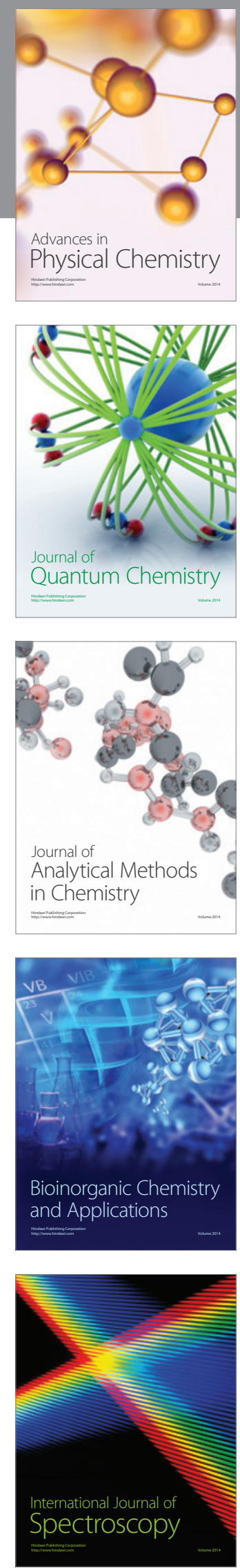